\title{
A fully automated binning method for improved SHARP reconstruction of free-breathing cardiac images
}

\author{
Aurelien Bustin ${ }^{1,2^{*}}$, Freddy Odille ${ }^{3,4}$, Guido P Kudielka ${ }^{1,3}$, Martin A Janich ${ }^{1}$, Anja C Brau ${ }^{5}$, Anne Menini ${ }^{1}$ \\ From 19th Annual SCMR Scientific Sessions \\ Los Angeles, CA, USA. 27-30 January 2016
}

\section{Background}

Despite recent progress in fast cardiac imaging, respiratory motion remains a challenging problem, usually leading to poor image quality when scanning poor breath-holder patients or acquiring high spatial resolution images. Today respiratory motion is compensated using navigators or external physiological sensors and can result in decreased scan efficiency and increased setup complexity. We recently proposed a motion compensated reconstruction, Single-sHot Accelerated Reconstruction with Preserved-features, or SHARP, that enables high-resolution motion-corrected reconstruction of multiple single-shot images acquired in free-breathing, with respiratory motion derived directly from the single-shot images. In the present work, a fast and automatic self-navigated binning method is described, which aims to accelerate the SHARP reconstruction process while improving image quality. The rationale for accelerating SHARP is that raw data acquired in similar motion states can be clustered into a reduced number of motion states, thereby, improving the quality of images from which to extract motion.

\section{Methods}

Accelerated single-shot cardiac-gated fast gradient echo imaging was performed on a 3T MR750w system (GE Healthcare, WI, USA) on four healthy volunteers during free breathing. Interleaved golden-ratio k-space acquisition pattern was used, as previously described. For comparison, a breath-hold scan was acquired with the same sequence. Low-resolution images were obtained from the k-space center of each shot and

${ }^{1}$ GE Global Research, Freising, Germany

Full list of author information is available at the end of the article were stacked together along the time dimension. The moving parts of the image were automatically extracted by band pass filtering along the time-dimension with the frequency interval $[0.05,0.5] \mathrm{Hz}$ corresponding to the frequency range of respiration. The motion mask was obtained by summing over time and thresholding the back-transformed volume. A singular value decomposition (SVD) was then applied on the 2D mask (Figure 1).

\section{Results}

The best fit line representing the temporal stack was the first column of the left-singular vectors which corresponds to the highest singular value. An estimate of the true respiratory signal is obtained from this vector. The algorithm is computationally inexpensive since it only requires filtering and SVD of a stack of $2 \mathrm{D}$ small patches. Now given the breathing signal, acquired data are summed according to similar position in the respiratory cycle, therefore reducing the amount of data shots (Figure 1). The recent SHARP reconstruction is applied to compute a motion-free image (Figure 2).

\section{Conclusions}

The proposed method allows the extraction of a respiratory signal directly from the acquired images, providing a way to reconstruct faster, higher-quality single-shot images in free-breathing without the need for navigators or external sensors. The next planned step is to apply the method to clinical applications, such as pediatric or severely ill patients, in which breath-hold requirement is challenging and yet high-quality, high-resolution imaging is still required. 

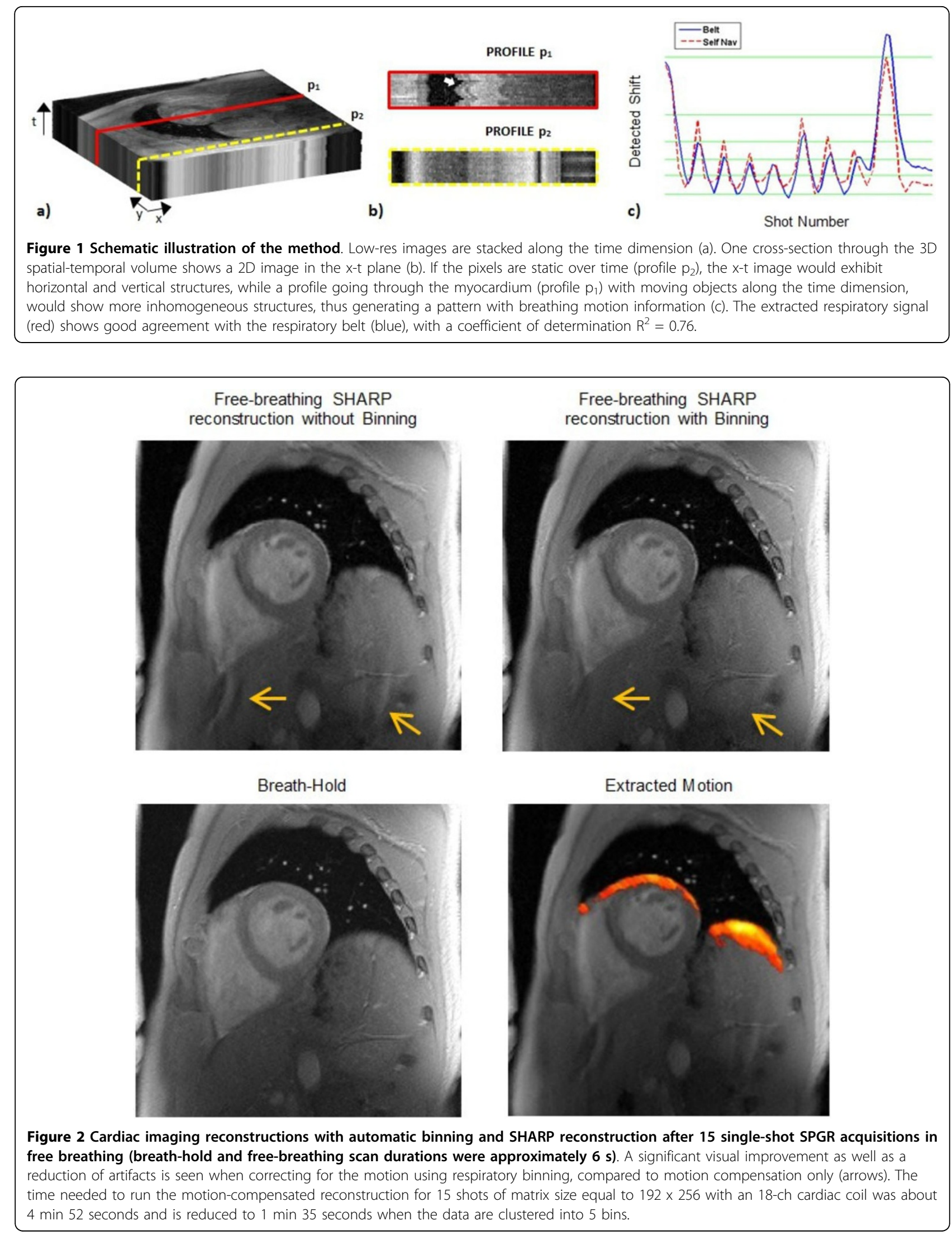


\section{Authors' details}

${ }^{1}$ GE Global Research, Freising, Germany. ${ }^{2}$ Department of Computer Science, Technische Universität München, München, Germany. ${ }^{3}$ Universite de Lorraine, Imagerie Adaptative Diagnostique et Interventionnelle, Nancy, France. ${ }^{4}$ Inserm, U947, Nancy, France. ${ }^{5}$ Cardiac Center of Excellence, GE Healthcare, Garching, Germany.

Published: 27 January 2016

doi:10.1186/1532-429X-18-S1-P267

Cite this article as: Bustin et al:: A fully automated binning method for improved SHARP reconstruction of free-breathing cardiac images. Journal of Cardiovascular Magnetic Resonance 2016 18(Suppl 1):P267.

Submit your next manuscript to BioMed Central and take full advantage of:

- Convenient online submission

- Thorough peer review

- No space constraints or color figure charges

- Immediate publication on acceptance

- Inclusion in PubMed, CAS, Scopus and Google Scholar

- Research which is freely available for redistribution

Submit your manuscript at www.biomedcentral.com/submit 